\title{
Spatial variability in the reproduction number of Ebola virus disease, Democratic Republic of the Congo, January-September 2019
}

Kenji Mizumoto ${ }^{1,2,3}$, Amna Tariq $^{3}$, Kimberlyn Roosa 3 , Jun Kong ${ }^{4,5,6}$, Ping Yan7, Gerardo Chowell ${ }^{3,8}$

1. Graduate School of Advanced Integrated Studies in Human Survivability, Kyoto University Yoshida-Nakaadachi-cho, Sakyo-ku, Kyoto, Japan

2. Hakubi Center for Advanced Research, Kyoto University, Yoshidahonmachi, Sakyo-ku, Kyoto, Japan

3. Department of Population Health Sciences, School of Public Health, Georgia State University, Atlanta, Georgia, United States of America

4. Department of Mathematics and Statistics, Georgia State University, Atlanta, Georgia, United States of America

5. Department of Computer Science, Georgia State University, Atlanta, Georgia, United States of America

6. Department of Computer Science, Emory University, Atlanta, Georgia, United States of America

7. Public Health Agency of Canada, Ottawa, Canada

8. Division of International Epidemiology and Population Studies, Fogarty International Center, National Institutes of Health, Bethesda, Maryland, United States of America

Correspondence: Kenji Mizumoto (mizumoto.kenji.5a@kyoto-u.ac.jp)

Mizumoto Kenji, Tariq Amna, Roosa Kimberlyn, Kong Jun, Yan Ping, Chowell Gerardo. Spatial variability in the reproduction number of Ebola virus disease,

Democratic Republic of the Congo, January-September 2019. Euro Surveill. 2019;24(42):pii=1900588. https://doi.org/10.2807/1560-7917.ES.2019.24.42.1900588

The ongoing Ebola virus disease epidemic (August 2018-October 2019) in the Democratic Republic of the Congo, has been exacerbated by deliberate attacks on healthcare workers despite vaccination efforts. Using a mathematical/statistical modelling framework, we present the quantified effective reproduction number (Rt) at national and regional levels as at 29 September. The weekly trend in Rt displays fluctuations while our recent national-level Rt falls slightly above 1.0 with substantial uncertainty, which suggests improvements in epidemic control.

Since its emergence in 1976, Ebola virus disease (EVD) has caused multiple outbreaks in several African countries, including the Democratic Republic of the Congo (DRC) [1]. The current epidemic in DRC that emerged in August 2018 contrasts with previous Ebola outbreaks in that transmission chains have persisted for over a year in a region of conflict, despite the availability of a highly effective vaccine [2]. Here, we seek to characterise the transmission potential of EVD and generate short-term forecasts at the national and health zone $(H Z)$ levels, focusing on the recent dynamics of the effective reproduction number $\left(R_{\mathrm{t}}\right)$. We also discuss our transmission estimates considering changes in surveillance indicators and frequency of outbreaks of violence.

\section{Current situation in the Democratic Republic of the Congo}

In terms of morbidity and mortality, the ongoing 2018-19 EVD outbreak in DRC was only surpassed in magnitude by the 2013-16 Western African epidemic [3]. Militia attacks and ethnic violence have been occurring in the affected region in DRC, which has fuelled a climate of community distrust of the government and of public health authorities resulting in fewer people seeking medical care for EVD [4,5]; healthcare centres and healthcare teams have been exclusively targeted undermining epidemiological surveillance efforts e.g. active case finding and isolation of infectious individuals, which are key for assessing the current state of the EVD epidemic and guiding public health efforts [5-7].

Ebola transmission hotspots in the DRC have varied geographically through the course of the outbreak [8]. At the beginning, the primary disease hotspots were centred in the $\mathrm{HZ}$ of Beni ( $\mathrm{HZ}$ 3), Mabalako $(\mathrm{HZ}$ 1), Mandima ( $\mathrm{HZ}$ 2), Butembo and Masereka HZ [5]. By December 2018, Katwa and Komanda HZs also exhibited intensified Ebola transmission [5]. The location of Ebola hotspots has been correlated with the frequency and location of outbreaks of violence and protests from community members that hinder the Ebola response efforts $[5,9,10]$.

\section{Epidemiological incidence cases}

Incidence curves of confirmed and probable cases of the ongoing Ebola epidemic in the DRC (August 2018October 2019) at the national and $\mathrm{HZ}$ levels are publicly available in weekly reports on the World Health Organization (WHO) website [5,9]. The latest national Ebola incidence curve by date of symptoms onset, was published on 8 October 2019 in Situation Report 62 [5]. 
At the $\mathrm{HZ}$ level, incidence curves were retrieved from the WHO Disease Outbreak News Report published on 10 October 2019 [9]. The date of reporting for the national incidence curve was defined as 6 October 2019 and the date of reporting for the HZ incidence curves was defined as 10 October 2019. Week of symptoms onset and week of reporting for each new Ebola case were obtained by analysing consecutive Ebola WHO reports (Situation Report 3-62) [5,9,11].

\section{Epidemiological modelling}

Let $f_{s}$ denote the probability mass function of the serial interval of EVD, where the serial interval is defined as the time from illness onset in the primary case to time of illness onset in the secondary case [12]. Then $f_{s}$, of length $s$ days, is given by:

$$
f_{s}=G(\mathrm{~s})-G(\mathrm{~s}-1) .
$$

For s>o, $G(s)$ represents the cumulative distribution function of the gamma distribution. We characterised the expected number of new incident cases $E\left[c_{i, t}\right]$ in $\mathrm{HZ} i$ at symptom onset week $t$ as follows:

$$
\mathrm{E}\left[c_{i, t}\right]=\sum_{j} r_{i j, t} \sum_{s=1}^{\infty} c_{j, t-s, f_{s}}
$$

where $r_{i j}$ denotes the average number of cases in $\mathrm{HZ} i$ infected by a single individual from $\mathrm{HZ} j$. Here we assume that the incidence, $c_{i, t}$, follows a Poisson sampling process with expected value $\mathrm{E}\left[c_{\mathrm{i}, \mathrm{t}}\right]$.

The reproduction matrix for seven geographic $\mathrm{HZs}$ is given by:

$$
\mathbf{M}_{t}=\left(\begin{array}{ccccccc}
r_{11, t} & r_{12} & r_{13} & r_{14} & r_{15} & r_{16} & r_{17} \\
r_{21} & r_{22, t} & r_{23} & r_{24} & r_{25} & r_{26} & r_{27} \\
r_{31} & r_{32} & r_{33, t} & r_{34} & r_{35} & r_{36} & r_{37} \\
r_{41} & r_{42} & r_{43} & r_{44, t} & r_{45} & r_{46} & r_{47} \\
r_{51} & r_{52} & r_{53} & r_{54} & r_{55, t} & r_{56} & r_{57} \\
r_{61} & r_{62} & r_{63} & r_{64} & r_{65} & r_{66, t} & r_{67} \\
r_{71} & r_{72} & r_{73} & r_{74} & r_{75} & r_{76} & r_{77, t}
\end{array}\right)
$$

This matrix is referred to as a next-generation matrix (NGM) in a fully susceptible population [13]. Using this matrix, we derive the instantaneous time-dependent effective reproduction number, $R_{\mathrm{t}}$, for the national transmission dynamics from the largest eigenvalue of the NGM. Under the assumption that the per-contact infection probability and the generation interval are consistent over time across HZs, the NGM quantifies the local (within zone) and inter-zone (across zones) patterns of transmission [14]. Then, the value of $R_{\mathrm{t}}$ for a specific $\mathrm{HZ} j$ is the sum of the local and inter-zone transmission (outgoing to other $\mathrm{HZs}$ ), or the sum of column $j$.
Local transmission (within-zone) dominates the overall transmission dynamics, so we estimate these rates as time-dependent parameters. Inter-zone transmission also contributes to the generation of secondary cases, but to a comparably smaller degree; thus, we model it as an invariant quantity for simplicity. The serial interval is characterised using a gamma distribution with the mean and standard deviation (SD) at 15.3 and 9.1 days, respectively [15]. We fixed the maximum value of the serial interval at 6 weeks at 0.99, which is the cumulative probability of the gamma distribution at 6 weeks.

Using the model calibrated to the epidemic data and the latest estimates of the reproduction matrix, we generated a 4-week forecast assuming that the $R_{t}$ remains stable throughout the period [16]. The forecast period is from week 39 to week 42 (30 September-27 October 2019). We estimated model parameters and made projections using a Monte Carlo Markov Chain (MCMC) method in a Bayesian framework. Point estimates and corresponding $95 \%$ credibility intervals (Crl) were drawn from the posterior probability distribution. All statistical analyses were done in $R$ version 3.5.2 ( $R$ Foundation for Statistical Computing, Vienna, Austria) and the 'rstan' package (No-U-Turn-Sampler (NUTS)).

\section{Findings from the real-time outbreak analysis}

Results indicate substantial spatial-temporal variation in $R_{\mathrm{t}}$ of Ebola virus across the seven HZs (Figure 1). The median effective reproduction number as of 8 October and the total number of new confirmed cases between 18 September-8 October 2019 by HZ are presented in Figure $1 \mathrm{~A}$ and $1 \mathrm{~B}$, respectively.

We estimated the reporting delay adjusted EVD incidence for each week $(t)$ at the national level and for each HZ from week 7 to week 38 (18 February-29 September 2019) (Figure 2) and the actual reported cases falls within the adjusted $\mathrm{Crl}$ for each $\mathrm{HZ}$ /national level.

We derived weekly estimates of the effective reproduction number, $R_{\mathrm{t}}$, for the national and $\mathrm{HZ}$ levels (Figure 3). We found that the overall national $R_{\mathrm{t}}$ lies well above the epidemic threshold of 1.0 for most of 2019 , with a multimodal pattern. After brief decline in July, our latest estimate of $R$ at the national level is 1.03 (95\% Crl: 0.59-2.12), indicating sustained transmission with substantial uncertainty straddling the epidemic threshold. Our estimates are also supported by the sensitivity analyses that examine the influence of small variations in the mean serial interval on our estimated $R_{\mathrm{t}}$ (Supplementary Figure $\mathrm{S} 1$ ).

Examining inter-zone transmission, where $r$ ii stands for the reproduction number from $\mathrm{HZ} j$ to $\mathrm{HZ} i$, the median value is 0.03 ( $95 \% \mathrm{Crl}: 0.00-0.12)$, with a maximum $r_{i j}$ value of 0.19 from Mambasa ( $\mathrm{HZ}$ 5) to Mandima ( $\mathrm{HZ}$ 2), and a minimum value of 0.00 from 
Geographical heterogeneity of Ebola virus disease reproduction number and confirmed cases across health zones, Democratic Republic of the Congo, 8 October 2019

\section{A. Median effective reproduction number as at 9 September 2019}

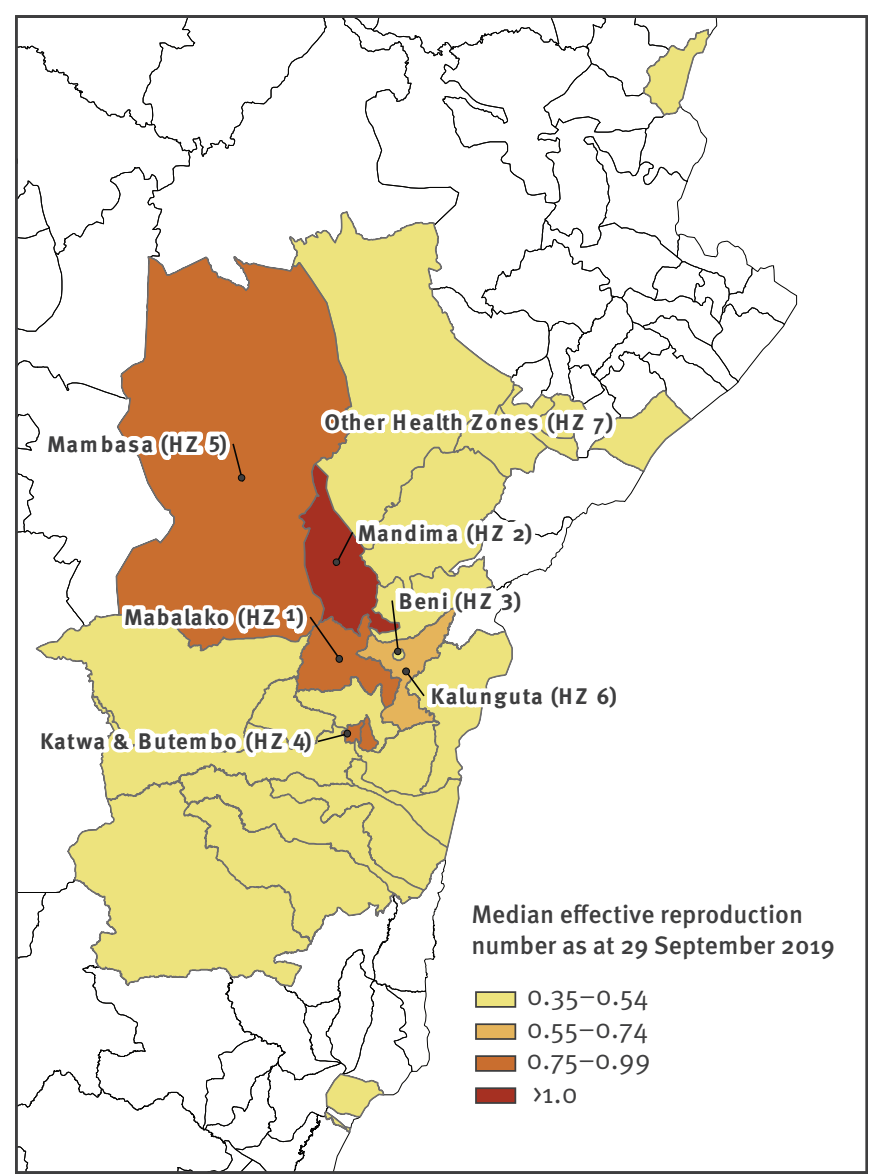

Katwa and Butembo ( $\mathrm{HZ}$ 4) to Mambasa ( $\mathrm{HZ}$ 5) (Figure 4). The sum of the outgoing $r_{\text {ij }}$ values for a given $\mathrm{HZ} j$ yields the combined inter-regional reproduction number from $\mathrm{HZ} j$ to each of the other $\mathrm{HZs}$. Mandima ( $\mathrm{HZ}$ 2) and Mambasa ( $\mathrm{HZ}$ 5) have notably higher outgoing transmission potential, with values of 0.30 and 0.45 , respectively (Figure 4 ). Including local transmission (within-zone) using the latest estimate of $R_{t}$, each column (or total $R_{t}$ ) corresponding with its respective $\mathrm{HZ}$ increases to $0.70,1.11,0.35,0.72,0.67,0.58$ and 0.35 from $\mathrm{HZ} 1$ to 7 , respectively.

Our short-term forecasts (week 39-week 42) are shown in Supplementary Figure S2. The predicted total number of cases from Mabalako $(\mathrm{HZ} 1)$ to Other $\mathrm{HZ}(\mathrm{HZ} 7)$ is estimated to be 6.5 (95\% Crl: 2.2-37.2), 31.2 (95\% Crl: 11.3-96.6), 9.6 (95\% Crl: 4.22-24.6), 11.4 (95\% Crl: 4.0-47.4), 3.5 (95\% Crl: 1.1-14.1), 8.5 (95\% Crl: 3.5-26.7) and 14.0 (95\% Crl: 6.5-30.1), respectively, while the predicted total number of cases across health zones is estimated at 95.2 (95\% Crl: 53.0-185.2).

\section{B. Confirmed cases 12 August-1 September 2019}

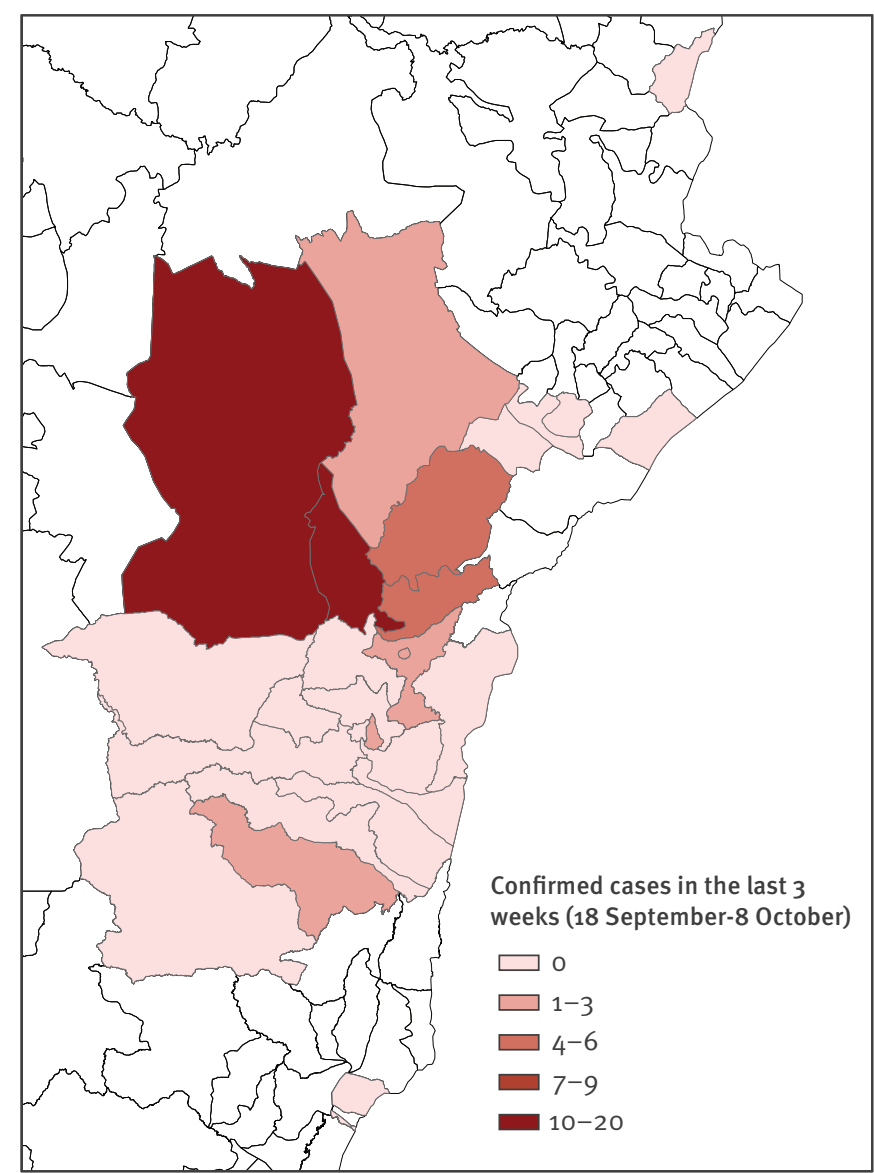

\section{Discussion}

This article to assesses the EVD transmission potential at the national and $\mathrm{HZ}$ levels through the dynamics of $R$ for the ongoing outbreak in the DRC, JanuarySeptember 2019. Our national monthly level estimates indicate an overall decreasing trend in mean $R_{\mathrm{t}}$ from 2.18 in July 2019 to 1.20 in September 2019. The recent national $R$, decline in July is consistent with changes in surveillance indicators, including a decline in the reporting delay from an average of 25.7 (Cl: $17.4^{-}$ 36.04) days in February 2019 to 11.02 ( $\mathrm{Cl}: 10.28-11.8)$ days in September 2019. Furthermore, there has been an improvement in contact tracing with almost $90 \%$ of the contacts being followed daily in September 2019 associated with the recent case decline in the region $[9,17,18]$.

Vaccination rates increased in the DRC by $47.9 \%$ from June 2019 to July 2019 among the people at risk for EVD, suggesting that public health measures have improved, supported by the gradual decline observed in EVD transmission. However, despite the improved control efforts, the frequency of violent attacks on healthcare 


\section{FIGURE 2}

Observed and estimated number of Ebola virus disease cases by health zone, Democratic Republic of the Congo, JanuarySeptember $2019(\mathrm{n}=2,498)$

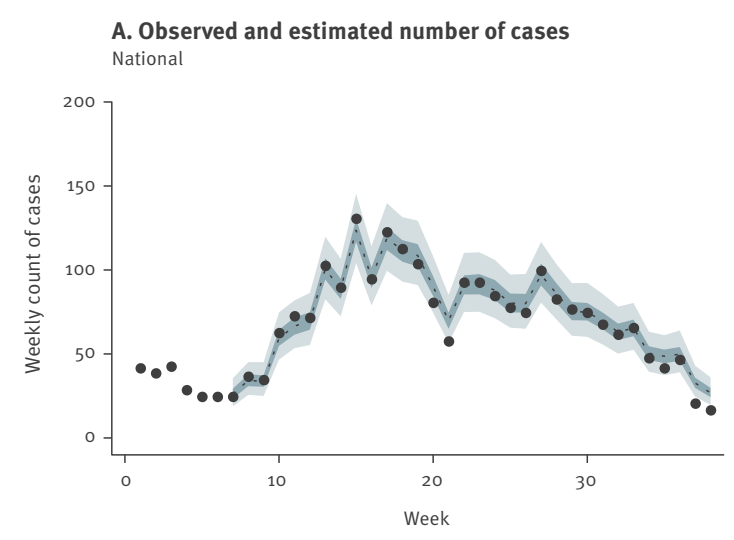

B. Observed and estimated number of cases Mabalako (HZ 1)

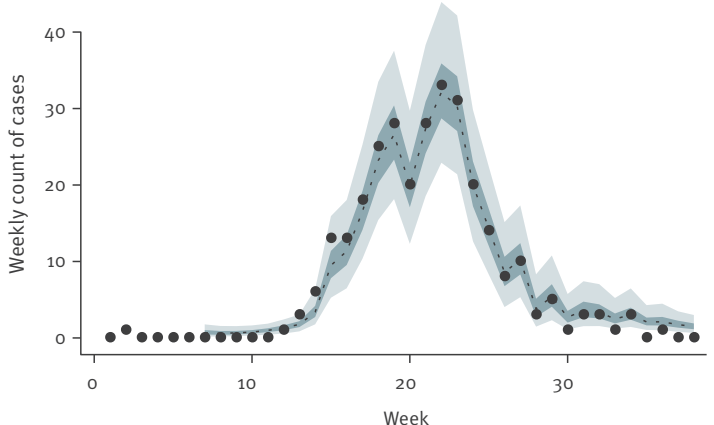

C. Observed and estimated number of cases Mandima ( $\mathrm{HZ}_{2}$ )

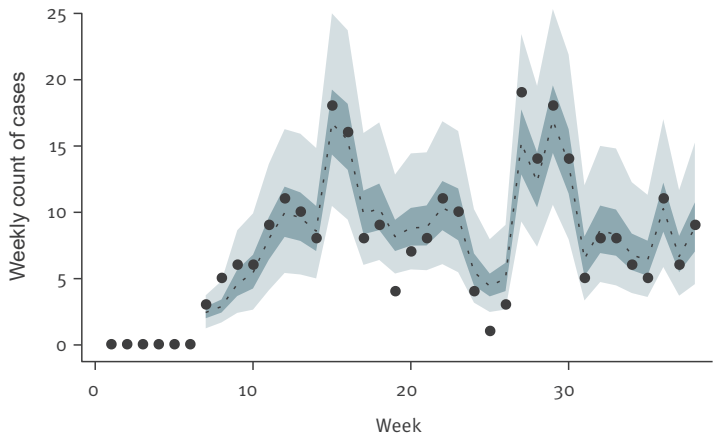

D. Observed and estimated number of cases Beni ( $\mathrm{HZ}_{3}$ )

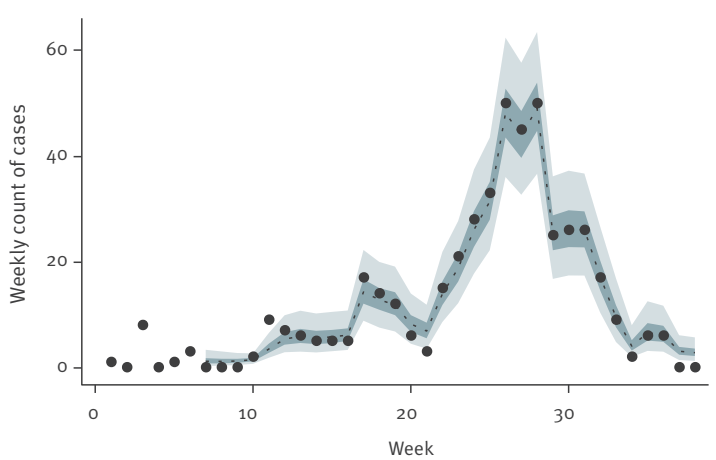

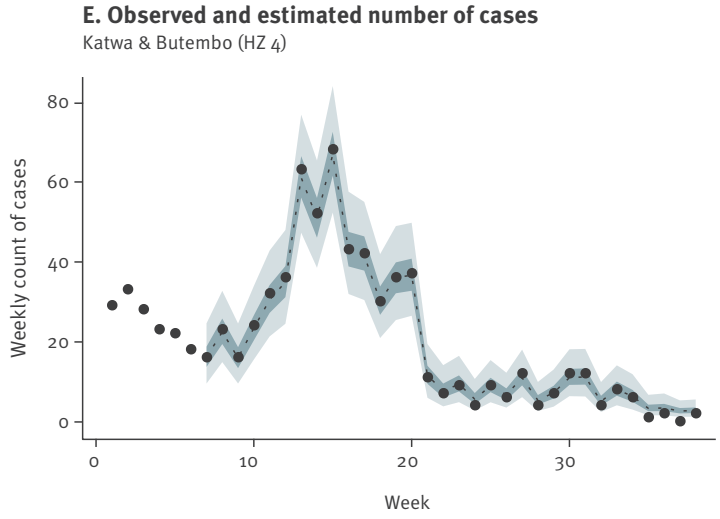

F. Observed and estimated number of cases Mambasa ( $\mathrm{HZ}_{5}$ )

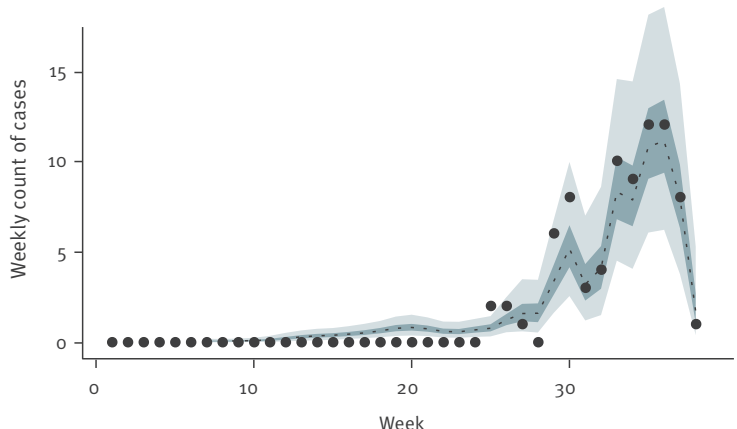

G. Observed and estimated number of cases Kalunguta (HZ 6)

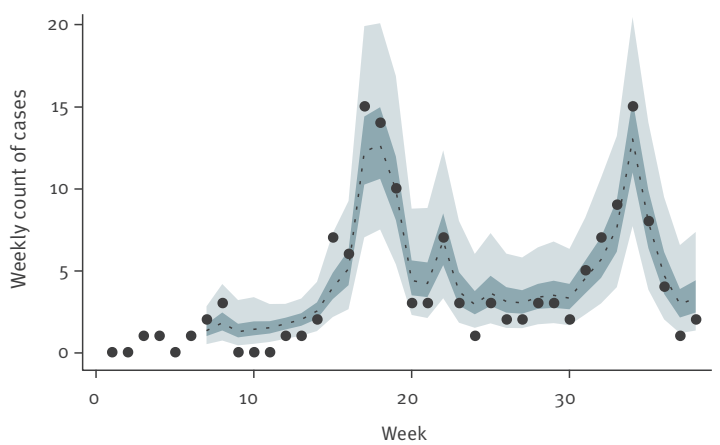

H. Observed and estimated number of cases Other Health Zones ( $\mathrm{HZ} 7$ )

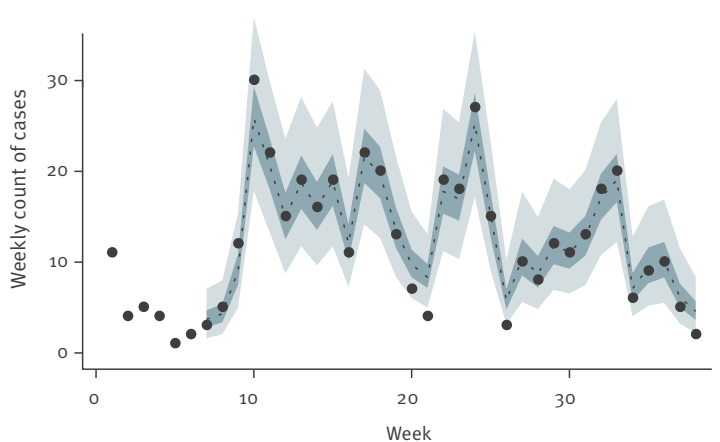

HZ: health zone.

Light and dark indicates $95 \%$ and 50\% credible intervals for posterior estimates, respectively. 


\section{FIGURE 3}

Time-dependent Ebola virus disease effective reproduction number by health zones, Democratic Republic of the Congo, January-September 2019
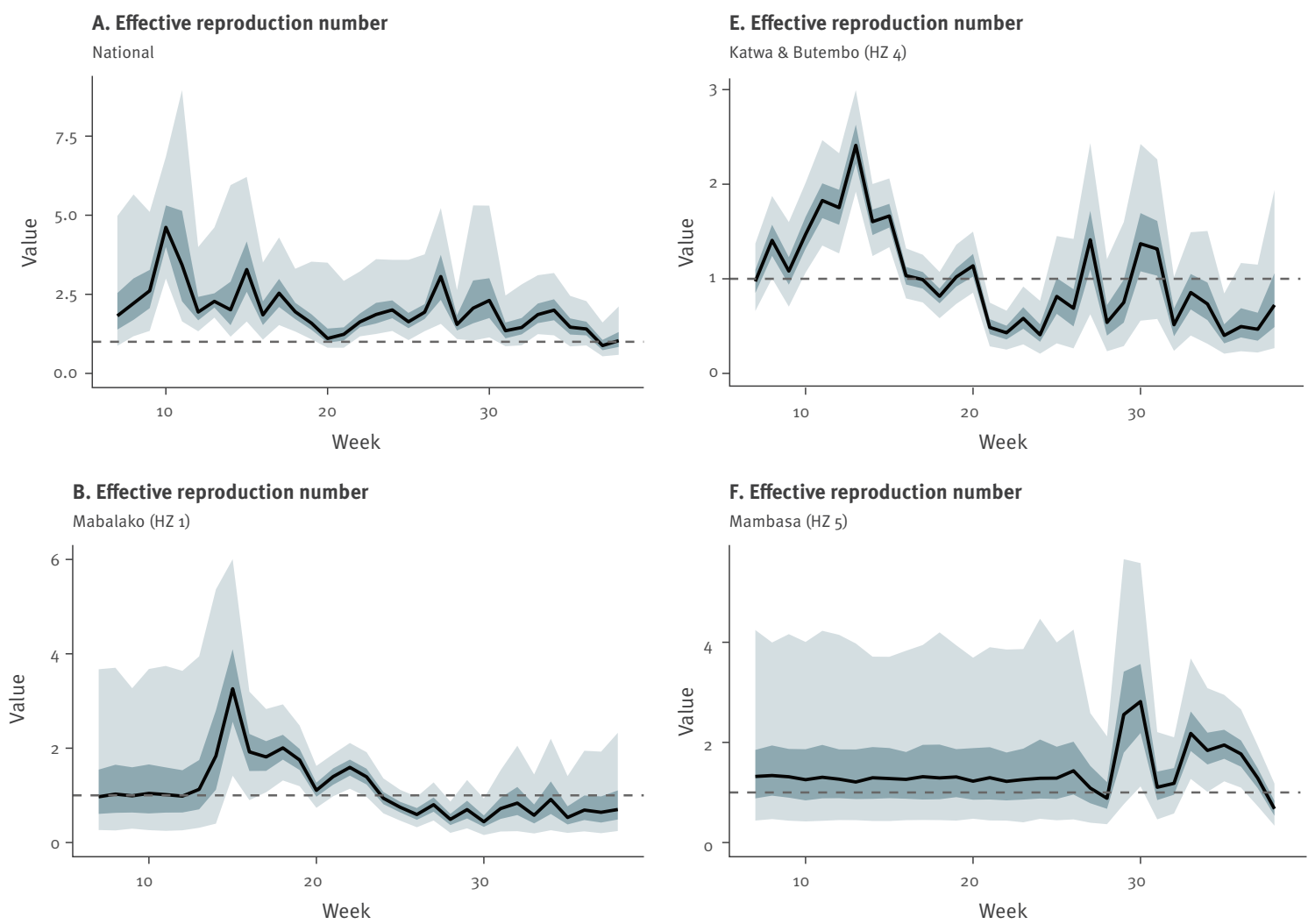

C. Effective reproduction number Mandima ( $\mathrm{HZ}_{2}$ )

G. Effective reproduction number Kalunguta (HZ 6)
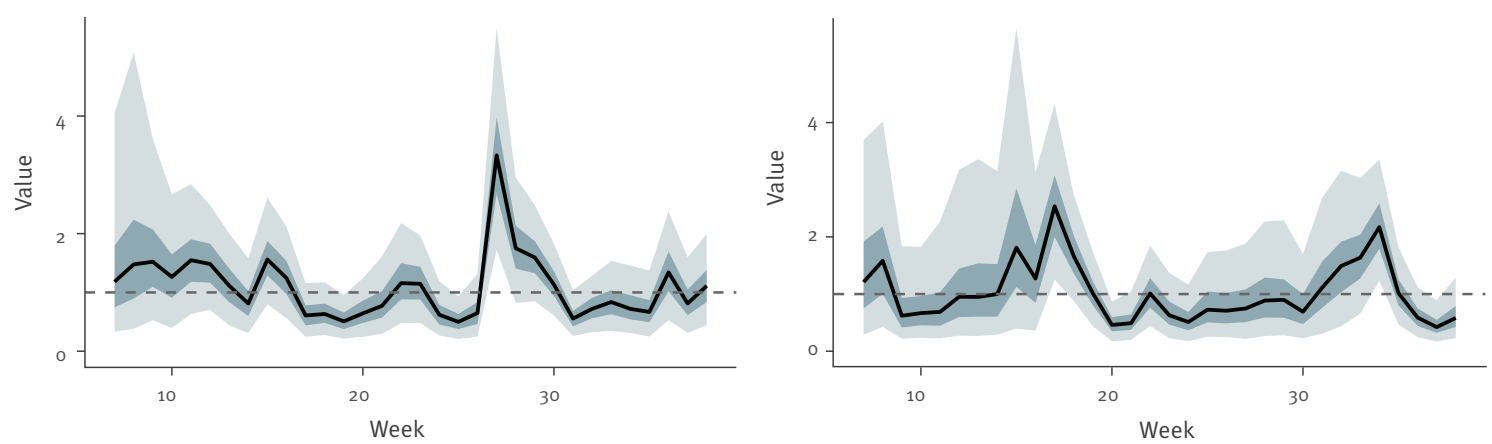

D. Effective reproduction number Beni $\left(\mathrm{HZ}_{3}\right)$
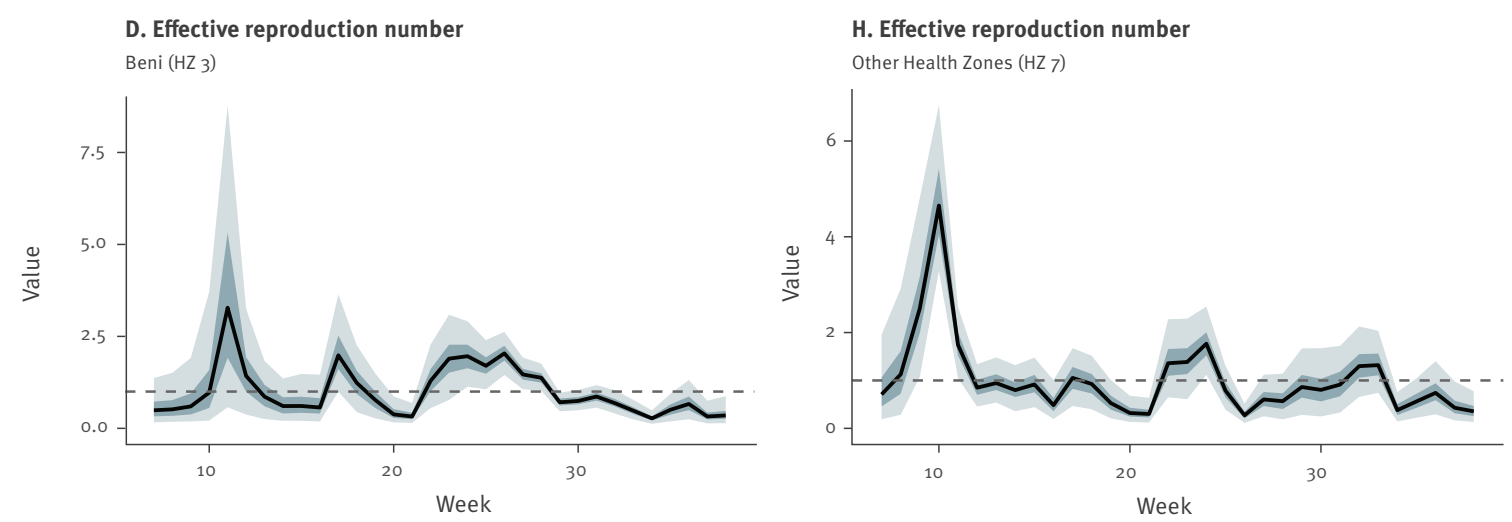

HZ: Health Zone.

The national effective reproduction number were calculated from the dominant eigenvalue of next-generation matrix. Light and dark indicates $95 \%$ and $50 \%$ credible intervals for posterior estimates, respectively. Horizontal grey dashed line shows the reproduction number at 1.0 for reference, below which the epidemic goes to decline. 


\section{FIGURE 4}

Inter-zone Ebola virus disease reproduction number matrix, Democratic Republic of the Congo, JanuarySeptember 2019

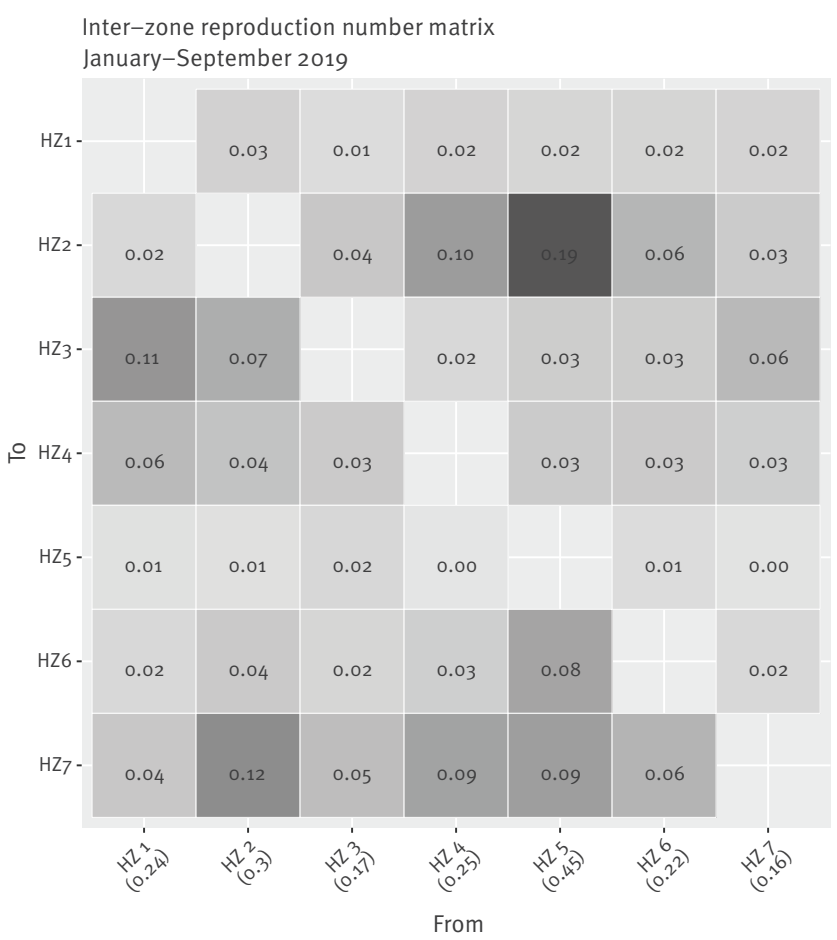

$\mathrm{HZ}$ : health zone.

$\mathrm{HZ}$ 1, 2, 3, 4, 5, 6 and 7 indicate Mabalak, Mandima, Beni, Katwa and Butembo, Kalunguta, and Other HZs, respectively. Number in the bottom line is the sum of the column, indicating the total transmission (within-zone and inter-zone transmission) by $\mathrm{HZ}$.

centres and healthcare teams has remained consistent with an average of five attacks per month in 2019; possibly influencing disease persistence and suboptimal diagnostic delays $[5,19]$. Increased EVD transmission from February-March 2019 (week 7-11) and from mid-June to mid-July 2019 (week 24-28) may be associated with documented attacks to response efforts in Katwa and Butembo ( $\left.\mathrm{HZ}_{4}\right)$ and in North Khivu (Figure 3) [1]. This highlights the need to further strengthen the Ebola control efforts by improving the security situation in the affected health zones. This will have a positive impact on the infection control practices in the affected areas and enhance community engagement in order to extinguish all transmission chains.

Our findings support spatial heterogeneity in transmission, with recent $R_{\mathrm{t}}$ estimates ranging from 0.4 in Beni ( $\mathrm{HZ} 3$ ) to 1.1 Mandima ( $\mathrm{HZ}$ 2). The transmission rates in Mandima ( $\mathrm{HZ}$ 2), where the epidemic originated, suggests case reintroduction and exportations within and across the $\mathrm{HZ}$ as potential contributing factors to the ongoing epidemic $[20,21]$. On the other hand, public mistrust in the health authorities has contributed to case resurgences in Beni ( $\mathrm{HZ}_{3}$ ), a region that reported 30 Ebola community deaths in July 2019. There are signs of gradual improvement in control efforts including active contact tracing and vaccinations, but such efforts could be further enhanced [5].

Our study has several limitations. While we relate the observed fluctuations in $R$ to outbreaks of violence, spatially-refined data would be required to explain the spatial variability in $R_{\mathrm{t}}$ over the course of the epidemic. Incorporating detailed epidemiological data (age, sex, etc.) as well as the timing, duration and intensity of public health efforts/disruptions into a mechanistic transmission model (or by conducting complementary spatial autoregressive modelling analyses) may allow the sources of spatial heterogeneity to be investigated [22]. We note that the inter-zone reproduction number for our analyses is taken as an 9-month average (January-September 2019) to facilitate the inference of the reproduction matrix. Further, Other $\mathrm{HZ}(\mathrm{HZ} 7)$ in our analyses comprised of several $\mathrm{HZs}$, for which we were not able to assess their transmission dynamics.

The $R_{\mathrm{t}}$ of the ongoing Ebola epidemic in DRC continues to display fluctuations with our most recent national estimate of $R_{t}$ reaching values slightly above the epidemic threshold of 1.0. Findings indicate that security incidents in the affected region continue to hamper the effectiveness of control interventions.

\section{Acknowledgements}

We thank Katsushi Kagaya for discussion on the model.

Funding statement: KM acknowledges support from the Japan Society for the Promotion of Science (JSPS) KAKENHI Grant Number 18K17368 and from the Leading Initiative for Excellent Young Researchers from the Ministry of Education, Culture, Sport, Science \& Technology of Japan. AT and KR support from a Georgia State University's Second Century Initiative $(2 \mathrm{Cl})$ doctoral fellowship. JK partly support from National Institute of Health $\mathrm{K}_{25} \mathrm{CA}_{181503}$, and U01CA242936.

GC acknowledges support from NSF grant 1414374 as part of the joint NSF-NIH-USDA Ecology and Evolution of Infectious Diseases program

\section{Conflict of interest}

None declared.

\section{Authors' contributions}

$\mathrm{KM}$ and $\mathrm{GC}$ conceived and designed the early study idea and built the model.

KM, AT, KR, JK, PY collected and processed data. KM and GC implemented statistical analysis. KM and AT wrote the first full draft. KM, AT, KR, PY and GC contributed to the interpretation of the results. KM, AT, KR, JK, PY and GC edited and commented on several earlier versions of the manuscript. GC provided supervision. 


\section{References}

1. Wannier SR, Worden L, Hoff NA, Amezcua E, Selo B, Sinai C, et al. Estimating the impact of violent events on transmission in Ebola virus disease outbreak, Democratic Republic of the Congo, 2018-2019. Epidemics. 2019;28:100353. https://doi. org/10.1016/j.epidem.2019.100353 PMID: 31378584

2. World Health Organization (WHO). Preliminary results on the efficacy of rVSV-ZEBOV-GP Ebola vaccine using the ring vaccination strategy in the control of an Ebola outbreak in the Democratic Republic of the Congo: an example of integration of research into epidemic response. Geneva: WHO; 2019. Available from: https://www.medbox.org/ context-facts-travel-advice/preliminary-results-on-theefficacy-of-rvsv-zebov-gp-ebola-vaccine-using-the-ringvaccination-strategy-in-the-control-of-anebola-outbreakin-the-democratic-republic-of-the-congo/toolboxes/ preview?

3. The Atlantic. Science. Yong E. The WHO Finally Sounds Its Loudest Alarm Over Ebola in the Congo, in The Atlantic. Online article. Available from: https://www.theatlantic.com/science/ archive/2019/07/the-who-finally-sounds-its-loudest-alarmover-the-congos-ebola-outbreak/594313/.

4. Nature International journal of medicine Maxmen A. Exclusive: Behind the front lines of the Ebola wars How the World Health Organization is battling bullets, politics and a deadly virus in the Democratic Republic of the Congo. Nature. 2019: Online article. Available from: https://www.nature.com/ articles/d41586-019-02673-7

5. World Health Organization (WHO). Situation Reports. Ebola health update - DRC, 2019, Situation Reports 2019. Geneva: WHO; 2019. Available from: https://www.who.int/emergencies/ diseases/ebola/drc-2019/situation-reports

6. Claude KM, Underschultz J, Hawkes MT. Ebola virus epidemic in war-torn eastern DR Congo. Lancet. 2018;392(10156):1399401. https://doi.org/10.1016/S0140-6736(18)32419-X PMID: 30297137

7. Medecins Sans Frontieres[Doctors without borders]. Ebola outbreak in Democratic Republic of Congo: Fighting an epidemic in a conflict zone. Article online. 2019. Available from: https://www.doctorswithoutborders.org/ ebola-outbreak-democratic-republic-congo

8. Majumder M, Rose S. Vaccine Deployment and Ebola Transmission Dynamics Estimation in Eastern DR Congo. SSRN. 2018.

9. World Health Organization (WHO). Emergencies preparedness, response Ebola virus disease Disease outbreak news. Geneva: WHO; 2019. Available from: https://who.int/csr/don/archive/ disease/ebola/en/

10. Ilunga Kalenga O, Moeti M, Sparrow A, Nguyen VK, Lucey D, Ghebreyesus TA. The Ongoing Ebola Epidemic in the Democratic Republic of Congo, 2018-2019. N Engl J Med. 2019;381(4):373-83. https://doi.org/10.1056/NEJMsr1904253 PMID: 31141654

11. World Health Organization (WHO). Ebola Virus Disease, Democratic Republic of the Congo, External Situation Reports. 2018.

12. Chowell G, Nishiura H. Transmission dynamics and control of Ebola virus disease (EVD): a review. BMC Med. 2014;12(1):196. https://doi.org/10.1186/s12916-014-0196-o PMID: 25300956

13. Diekmann O, Heesterbeek JAP, Roberts MG. The construction of next-generation matrices for compartmental epidemic models. J R Soc Interface. 2010;7(47):873-85. https://doi.org/10.1098/ rsif.2009.0386 PMID: 19892718

14. Nishiura H, Chowell G. Early transmission dynamics of Ebola virus disease (EVD), West Africa, March to August 2014. Euro Surveill. 2014;19(36):20894. https://doi.org/10.2807/15607917.ES2014.19.36.20894 PMID: 25232919

15. Barry A, Ahuka-Mundeke S, Ali Ahmed Y, Allarangar Y, Anoko J, Archer BN, et al. Ebola Outbreak Epidemiology Team. Outbreak of Ebola virus disease in the Democratic Republic of the Congo, April-May, 2018: an epidemiological study. Lancet. 2018;392(10143):213-21. https://doi.org/10.1016/So1406736(18)31387-4 PMID: 30047375

16. Garske T, Cori A, Ariyarajah A, Blake IM, Dorigatti I, Eckmanns $\mathrm{T}$, et al. Heterogeneities in the case fatality ratio in the West African Ebola outbreak 2013-2016. Philos Trans R Soc Lond B Biol Sci. 2017;372(1721):20160308. PMID: 28396479

17. World Health Organization (WHO). Ebola Virus Disease, Democratic Republic of the Congo, Situational Report 61. Geneva: WHO; Available from: https://www.who.int/ publications-detail/ebola-virus-disease-democratic-republicof-congo-external-situation-report-61-2019

18. Tariq A, Roosa K, Mizumoto K, Chowell G. Assessing reporting delays and the effective reproduction number: The Ebola epidemic in DRC, May 2018-January 2019. Epidemics.
2019;26:128-33. https://doi.org/10.1016/j.epidem.2019.01.003 PMID: 30880169

19. Insight I. Attacks on Ebola Response Information Alert. 2019 [Accessed Sep 10 2019]; Available from: https://mailchi. $\mathrm{mp} / 5 \mathrm{a} 131 \mathrm{cfo} 4 \mathrm{ad}$ /information-alert-1-attacks-on-ebolaresponse-1813529? $=7434253 \mathrm{cfb}$.

20. Centers for Disease Control and Prevention (CDC). Eradicating Ebola: Building on Lessons Learned and Medical Advancements. Atlanta: CDC; 2019. Available from: https:// www.cdc.gov/washington/testimony/2019/t20190604.htm.

21. Center for Infectious Disease Research and Policy (CIDRAP). Soucheray S. Ebola persists in Beni and Mandima hot spots; orphan numbers grow. Minnesota: CIDRAP; 2019. Available from: http://www.cidrap.umn.edu/news-perspective/2019/08/ ebola-persists-beni-and-mandima-hot-spots-orphan-numbersgrow

22. Mizumoto K, Kobayashi T, Chowell G. Transmission potential of modified measles during an outbreak, Japan, MarchMay 2018. Euro Surveill. 2018;23(24):1800239. https:// doi.org/10.2807/1560-7917.ES.2018.23.24.1800239 PMID: 29921344

\section{License, supplementary material and copyright}

This is an open-access article distributed under the terms of the Creative Commons Attribution (CC BY 4.0) Licence. You may share and adapt the material, but must give appropriate credit to the source, provide a link to the licence and indicate if changes were made.

Any supplementary material referenced in the article can be found in the online version.

This article is copyright of the authors or their affiliated institutions, 2019. 